PO-TH 14/O5

USM-TH-323

\title{
Fermion masses and mixings in an $S U(5)$ grand unified model with an extra flavor symmetry
}

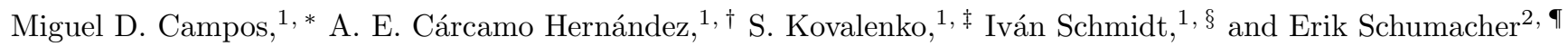 \\ ${ }^{1}$ Universidad Técnica Federico Santa María and Centro Científico-Tecnológico de Valparaíso \\ Casilla 110-V, Valparaíso, Chile \\ ${ }^{2}$ Fakultät für Physik, Technische Universität Dortmund \\ D-44221 Dortmund, Germany
}

\begin{abstract}
We propose a model based on the $S U(5)$ grand unification with an extra $A_{4} \otimes Z_{2} \otimes Z_{2}^{\prime} \otimes Z_{2}^{\prime \prime} \otimes U(1)_{f}$ flavor symmetry, which accounts for the pattern of the SM fermion masses and mixings. The observed hierarchy of charged fermion masses and quark mixing matrix elements arises from a generalized Froggatt-Nielsen mechanism triggered by a scalar $\mathbf{2 4}$ representation of $S U(5)$ charged under the global $U(1)_{f}$ and acquiring a VEV at the GUT scale. The light neutrino masses are generated via a radiative seesaw mechanism with a single heavy Majorana neutrino and neutral scalars running in the loops. The model predictions for both quark and lepton sectors are in good agreement with the experimental data. The model predicts an effective Majorana neutrino mass, relevant for neutrinoless double beta decay, with values $m_{\beta \beta}=4$ and $50 \mathrm{meV}$ for the normal and the inverted neutrino spectrum, respectively. The model also features a suppression of CP violation in neutrino oscillations, a low scale for the heavy Majorana neutrino (few $\mathrm{TeV}$ ) and, due to the unbroken $Z_{2}$ symmetry, a natural dark matter candidate.
\end{abstract}

\section{INTRODUCTION}

The great success of the Standard Model (SM) in the description of electroweak phenomena, recently confirmed with the LHC discovery of the Higgs boson, nevertheless leaves many unresolved problems. Among the most pressing are the smallness of neutrino masses, the puzzling pattern of fermion masses and mixings, and the existence of the three families of quarks and leptons. In the search for a solution of these problems various extensions of the SM with additional flavor symmetries have been proposed in the literature (for a review see, e.g., Refs. [1-3]). Historically, some of these symmetries were hinted at by the tribimaximal (TBM) ansatz for the leptonic mixing matrix,

$$
U_{\mathrm{TBM}}=\left[\begin{array}{ccc}
\sqrt{\frac{2}{3}} & \frac{1}{\sqrt{3}} & 0 \\
-\frac{1}{\sqrt{6}} & \frac{1}{\sqrt{3}} & -\frac{1}{\sqrt{2}} \\
-\frac{1}{\sqrt{6}} & \frac{1}{\sqrt{3}} & \frac{1}{\sqrt{2}}
\end{array}\right] .
$$

leading to the neutrino mixing angles $\left(\sin ^{2} \theta_{12}\right)_{\mathrm{TBM}}=1 / 3,\left(\sin ^{2} \theta_{23}\right)_{\mathrm{TBM}}=1 / 2$ and $\left(\sin ^{2} \theta_{13}\right)_{\mathrm{TBM}}=0$. However, recent measurements of a nonzero value of the reactor mixing angle $\theta_{13}$ by the Daya Bay [4], T2K [5], MINOS [6], Double CHOOZ [7] and RENO [8] have already ruled out the exact TBM pattern, as shown in Tables [] and (based on Ref. [9]) for the normal $(\mathrm{NH})$ and inverted $(\mathrm{IH})$ hierarchies of the neutrino mass spectrum. Nevertheless, the smallness of the reactor angle still allows for the TBM to serve as a first-order approximation in the construction of realistic models of lepton mixing based on flavor symmetries.

\footnotetext{
*Electronic address: miguel.campos@postgrado.usm.cl

${ }^{\dagger}$ Electronic address: antonio.carcamo@usm.cl

‡Electronic address: sergey.kovalenko@usm.cl

$\S$ Electronic address: ivan.schmidt@usm.cl

IElectronic address: erik.schumacher@tu-dortmund.de
} 


\begin{tabular}{|c|c|c|c|c|c|}
\hline Parameter & $\Delta m_{21}^{2}\left(10^{-5} \mathrm{eV}^{2}\right)$ & $\Delta m_{31}^{2}\left(10^{-3} \mathrm{eV}^{2}\right)$ & $\left(\sin ^{2} \theta_{12}\right)_{\exp }$ & $\left(\sin ^{2} \theta_{23}\right)_{\exp }$ & $\left(\sin ^{2} \theta_{13}\right)_{\exp }$ \\
\hline Best fit & 7.62 & 2.55 & 0.320 & 0.613 & 0.0246 \\
\hline $1 \sigma$ range & $7.43-7.81$ & $2.46-2.61$ & $0.303-0.336$ & $0.573-0.635$ & $0.0218-0.0275$ \\
\hline $2 \sigma$ range & $7.27-8.01$ & $2.38-2.68$ & $0.29-0.35$ & $0.38-0.66$ & $0.019-0.030$ \\
\hline $3 \sigma$ range & $7.12-8.20$ & $2.31-2.74$ & $0.27-0.37$ & $0.36-0.68$ & \\
\hline
\end{tabular}

Table I: Range for experimental values of neutrino mass squared splittings and leptonic mixing parameters, taken from Ref. [9], for the case of normal hierarchy.

\begin{tabular}{|c|c|c|c|c|c|}
\hline Parameter & $\Delta m_{21}^{2}\left(10^{-5} \mathrm{eV}^{2}\right)$ & $\Delta m_{13}^{2}\left(10^{-3} \mathrm{eV}^{2}\right)$ & $\left(\sin ^{2} \theta_{12}\right)_{\exp }$ & $\left(\sin ^{2} \theta_{23}\right)_{\exp }$ & $\left(\sin ^{2} \theta_{13}\right)_{\exp }$ \\
\hline Best fit & 7.62 & 2.43 & 0.320 & 0.600 & 0.0250 \\
\hline $1 \sigma$ range & $7.43-7.81$ & $2.37-2.50$ & $0.303-0.336$ & $0.569-0.626$ & $0.0223-0.0276$ \\
\hline $2 \sigma$ range & $7.27-8.01$ & $2.29-2.58$ & $0.29-0.35$ & $0.39-0.65$ & $0.020-0.030$ \\
\hline $3 \sigma$ range & $7.12-8.20$ & $2.21-2.64$ & $0.27-0.37$ & $0.37-0.67$ & $0.017-0.033$ \\
\hline
\end{tabular}

Table II: Range for experimental values of neutrino mass squared splittings and leptonic mixing parameters, taken from Ref. [9], for the case of inverted hierarchy.

Since the mixing patterns of leptons and quarks are significantly different, it is challenging to implement a unique symmetry, able to describe the small quark mixing angles and the (two) large leptonic ones at the same time.

Grand unified theories (GUTs), endowed with global flavor symmetries, may be an appropriate setup for a unified description of the masses and mixings of leptons and quarks. This is motivated by the fact that leptons and quarks are members of the same multiplets of the GUT group, which relates their masses and mixings. 10, 11]. Various GUT models with flavor symmetries have been proposed in the literature [12 22]. For a general review see for example [23, 24].

In this paper we propose a version of the $S U(5)$ GUT model with an additional global flavor symmetry group $A_{4} \times Z_{2} \times Z_{2}^{\prime} \times Z_{2}^{\prime \prime} \times U(1)_{f}$. It involves a horizontal symmetry $U_{f}(1)$, allowing us to naturally introduce the fermion mass hierarchies through a generalized Froggatt-Nielsen mechanism [25]. The discrete symmetry groups $A_{4}$ and three different $Z_{2}$ are needed in order to reproduce the specific patterns of mass matrices in the quark and lepton sectors.

The embedding of the model in a nonminimal $S U(5)$ GUT requires a significant extension of the scalar sector. The particular role of each additional scalar field and the corresponding particle assignments under the symmetry group of the model are explained in details in Sec. [I] On the other hand, in analogy to Ref. [26], we consider only one additional right-handed neutrino $N_{R}$ in order to explain the masses and mixings in the neutrino sector. The light neutrino masses are generated in our model through a radiative seesaw mechanism, in which neutrinos receive their masses only from radiative corrections at one-loop level. The smallness of the neutrino masses is a natural consequence of the small one-loop contributions and the quadratic dependence on the neutrino Yukawa couplings. In contrast to the regular seesaw type I scenarios, the mass of the right-handed neutrino can therefore be kept at the TeV scale. For a general review of the radiative seesaw we refer readers, for example, to Ref. [27], and to Ref. 28] for its discussion in the context of flavor symmetries.

Our model describes a realistic pattern of the SM fermion masses and mixings. The model has 14 free effective parameters, which allow us to reproduce the experimental values of 18 observables, i.e., 9 charged fermion masses, 2 neutrino mass squared splittings, 3 lepton mixing parameters and 4 parameters of the Wolfenstein parametrization of the CKM quark mixing matrix. Let us note that the similar model of Ref. [19], with an $S U(5)$ GUT supersymmetric setup and flavor symmetries, has 14 free effective parameters aimed at reproducing the above mentioned 18 observables. The paper is organized as follows. In Sec. [I] we outline the proposed model. In Sec. III we present our results regarding neutrino masses and mixing, which is followed by a numerical analysis. Our results for the quark sector, with the corresponding numerical analysis, are presented in Sec. IV] We conclude with discussions and a summary in Sec. V Some necessary facts about the $A_{4}$ group are collected in Appendix $\mathrm{A}$.

\section{THE MODEL}

As is well known, the minimal $S U(5)$ GUT [29] with fermions in $\overline{\mathbf{5}}+\mathbf{1 0}$ and the scalars in $\mathbf{5}+\mathbf{2 4}$ representations of $S U(5)$, suffers from various problems. In particular, it predicts wrong relations between the down-type quark 
and charged lepton masses, short proton life-time, and the unification of gauge couplings does not agree with the values of $\alpha_{S}, \sin \theta_{W}$ and $\alpha_{e m}$ at the $M_{Z}$ scale. There is no place in the minimal model for a nonzero neutrino mass, in contradiction with the neutrino oscillation experiments. Some of these problems can be solved by an extension of the model field content including, in particular, a scalar 45 representation of $S U(5)$ 30 42]. However, in this next-to-minimal $S U(5)$ GUT the hierarchy among the fermion masses is not understood and translates to an unexplained hierarchy among the different Yukawa couplings. This motivates implementing a generalized FroggatNielsen mechanism, where the fermion mass hierarchy is explained by a spontaneously broken group $U(1)_{f}$ with a special $U(1)_{f}$ charge assignment to the fields participating in the Yukawa terms. Our model is a multi-Higgs extension of the next-to-minimal $S U(5) \mathrm{GUT}$, and the full symmetry $\mathcal{G}$ is broken in two subsequent steps:

$$
\begin{gathered}
\mathcal{G}=S U(5) \otimes A_{4} \otimes Z_{2} \otimes Z_{2}^{\prime} \otimes Z_{2}^{\prime \prime} \otimes U(1)_{f} \\
\Downarrow \Lambda_{G U T} \\
S U(3)_{C} \otimes S U(2)_{L} \otimes U(1)_{Y} \otimes Z_{2} \\
\Downarrow \Lambda_{E W} \\
S U(3)_{C} \otimes U(1)_{e m} \otimes Z_{2}
\end{gathered}
$$

The discrete non-Abelian tetrahedral symmetry group $A_{4}$, the group of even permutations of four objects, is the smallest group with one three-dimensional and three distinct one-dimensional irreducible representations (irreps), naturally accommodating the three families of fermions. In the literature this group has been extensively studied in the context of the flavor problem and neutrino physics (cf. [28, 43, 44]). The role of the other symmetry group factors of $\mathcal{G}$ will be explained in what follows.

In the present model the fermion sector is extended by introducing only one additional field, a Majorana neutrino $N_{R}$ which is a singlet under the SM group. The three families of left- and right-handed fermions, corresponding to the $\overline{\mathbf{5}}$ irrep of $S U(5)$, are unified into an $A_{4}$ triplet in order to have one Yukawa term for the interaction with the righthanded neutrino $N_{R}$, analogously to Ref. [26]. The three families of left- and right-handed fermions accomodated into a 10 irrep of $S U(5)$ are assigned to the three different $A_{4}$ singlets $\mathbf{1}, \mathbf{1}^{\prime}, \mathbf{1}^{\prime \prime}$. The only right-handed SM singlet neutrino $N_{R}$ of our model is assigned to the $\mathbf{1}$ of $A_{4}$ in order for its Majorana mass term be invariant under this symmetry. The presence of this term is crucial for our construction, as explained below. Note that neither the $\mathbf{1}^{\prime}$ nor $\mathbf{1}^{\prime \prime}$ singlet representations of $A_{4}$ satisfy this condition, as can be seen from the multiplication rules in Eq. (A1). The fermion assignments under the group $\mathcal{G}=S U(5) \otimes A_{4} \otimes Z_{2} \otimes Z_{2}^{\prime} \otimes Z_{2}^{\prime \prime} \otimes U(1)_{f}$ are

$$
\begin{gathered}
\psi^{i}=\left(\psi^{i(1)}, \psi^{i(2)}, \psi^{i(3)}\right) \sim\left(\overline{\mathbf{5}}, \mathbf{3}, 1,1,-1, Q_{\overline{\mathbf{5}}}^{(\psi)}\right), \quad N_{R} \sim(\mathbf{1}, \mathbf{1},-1,1,-1,0) . \\
\Psi_{i j}^{(1)} \sim\left(\mathbf{1 0}, \mathbf{1}, 1,1,1, Q_{\mathbf{1 0}}^{(1)}\right), \quad \Psi_{i j}^{(2)} \sim\left(\mathbf{1 0}, \mathbf{1}^{\prime}, 1,1,1, Q_{\mathbf{1 0}}^{(2)}\right), \quad \Psi_{i j}^{(3)} \sim\left(\mathbf{1 0}, \mathbf{1}^{\prime \prime}, 1,1,1, Q_{\mathbf{1 0}}^{(3)}\right), \quad i, j=1,2,3,4,5 .
\end{gathered}
$$

More explicitly, the fermions are accommodated as [45]

$$
\begin{gathered}
\Psi_{i j}^{(f)}=\frac{1}{\sqrt{2}}\left(\begin{array}{ccccc}
0 & u_{3}^{(f) c} & -u_{2}^{(f) c} & -u_{1}^{(f)} & -d_{1}^{(f)} \\
-u_{3}^{(f) c} & 0 & u_{1}^{(f) c} & -u_{2}^{(f)} & -d_{2}^{(f)} \\
u_{2}^{(f) c} & -u_{1}^{(f) c} & 0 & -u_{3}^{(f)} & -d_{3}^{(f)} \\
u_{1}^{(f)} & u_{2}^{(f)} & u_{3}^{(f)} & 0 & -l^{(f) c} \\
d_{1}^{(f)} & d_{2}^{(f)} & d_{3}^{(f)} & l^{(f) c} & 0
\end{array}\right)_{L} \quad \text { } \quad i, j=1,2,3,4,5 . \\
\psi^{i(f)}=\left(d_{1}^{(f) c}, d_{2}^{(f) c}, d_{3}^{(f) c}, l^{(f)},-\nu_{f}\right)_{L} .
\end{gathered}
$$

Here the subscripts correspond to the different quark colors, while the superscript $f$ refers to fermion families.

The scalar sector is composed of the following $S U(5)$ representations: one 24, one 45, seven 5's and six 1's. One set of three 5's and the two sets of $S U(5)$ singlets are unified into three $A_{4}$ triplets. The remaining scalar fields, i.e., one $\mathbf{4 5}$, one $\mathbf{2 4}$ and the remaining set of the four $\mathbf{5}$ 's, are accommodated by two trivial and two different nontrivial $A_{4}$ singlets. Thus the $\mathcal{G}$ assignments of the scalar fields of our model are

$$
\chi=\left(\chi_{1}, \chi_{2}, \chi_{3}\right) \sim(\mathbf{1}, \mathbf{3}, 1,-1,1,0), \quad \xi=\left(\xi_{1}, \xi_{2}, \xi_{3}\right) \sim\left(\mathbf{1}, \mathbf{3}, 1,1,1, Q_{1}^{(\xi)}\right),
$$




$$
\begin{gathered}
S_{i}=\left(S_{i}^{(1)}, S_{i}^{(2)}, S_{i}^{(3)}\right) \sim\left(\mathbf{5}, \mathbf{3},-1,1,1, Q_{\mathbf{5}}^{(S)}\right), \\
H_{i}^{(1)} \sim\left(\mathbf{5}, \mathbf{1}, 1,1,-1, Q_{\mathbf{5}}^{(1)}\right), \quad H_{i}^{(2)} \sim\left(\mathbf{5}, \mathbf{1}^{\prime}, 1,1,1, Q_{\mathbf{5}}^{(2)}\right), \quad H_{i}^{(3)} \sim\left(\mathbf{5}, \mathbf{1}^{\prime \prime}, 1,1,1, Q_{\mathbf{5}}^{(3)}\right), \\
H_{i}^{(4)} \sim\left(\mathbf{5}, \mathbf{1}, 1,1,1, Q_{\mathbf{5}}^{(4)}\right), \quad \Sigma_{j}^{i} \sim\left(\mathbf{2 4}, \mathbf{1}, 1,-1,1,-\frac{1}{2}\right), \quad \Phi_{j k}^{i} \sim\left(\mathbf{4 5}, \mathbf{1}, 1,1,-1, Q_{\mathbf{4 5}}^{(\Phi)}\right) .
\end{gathered}
$$

We introduce two sets of $A_{4}$ triplets $S U(5)$ singlets in order to separate the interactions responsible for the light neutrino masses from those that generate the down-type quark and charged lepton masses. The $A_{4}$ triplet $S U(5)$ singlet $\chi$ is the only set of scalars which is neutral under the $U(1)_{f}$ symmetry, while the remaining scalars have nontrivial $U(1)_{f}$ charges. Notice that the two sets of 5's, i.e., $H_{i}^{(h)}(h=1,2,3,4)$ and $S_{i}^{(f)}(f=1,2,3)$ have different $Z_{2}$ parities. With respect to the fermion sector, only the three families of fermions, corresponding to the $\overline{\mathbf{5}}$ irrep of $S U$ (5), are unified into an $A_{4}$ triplet. Besides that, the three families of fermions embedded into the 10 irrep of $S U(5)$ are assigned to three different $A_{4}$ singlets, i.e, $\mathbf{1}, \mathbf{1}^{\prime}, \mathbf{1}^{\prime \prime}$. Then, in order to build the required Yukawa interactions for charged fermions, we need the following scalars: four 5's assigned to $A_{4}$ singlets (two of them assigned to a $A_{4}$ trivial singlets and the other ones assigned to $A_{4}$ nontrivial singlets), one $\mathbf{4 5}$ assumed to be a trivial $A_{4}$ singlet $\mathbf{1}$, the $S U(5)$ singlet $A_{4}$ triplet $\xi$ and the scalar field $\Sigma$ in the $\mathbf{2 4}$ representation of $S U(5)$. As previously mentioned, having scalar fields in the 45 representation of $S U(5)$ is crucial in order to get the correct mass relations of down-type quarks and charged leptons. Concerning the breakdown of the group $\mathcal{G}$ in Eq. (2), the scalar field $\Sigma$ is needed to trigger the generalized Froggatt-Nielsen mechanism responsible for generating the masses of charged fermions via higher dimensional Yukawa terms. Besides that, the scalar field $\Sigma$ acquires a vacuum expectation value (VEV) at the GUT scale $\Lambda_{G U T}=10^{16} \mathrm{GeV}$ and triggers the first step of symmetry breaking in Eq. (2). This first step is also induced by the $A_{4}$ scalar triplet $\xi$ acquiring a VEV at the GUT scale. The second step of symmetry breaking, is due to the scalars $H_{i}^{(h)}(h=1,2,3,4)$ and $\Phi_{j k}^{i}$ acquiring VEVs at the electroweak scale. The scalar $\chi$, being an $S U(5)$ singlet, may receive its VEV at any scale below $\Lambda_{G U T}$, in particular around TeV. The four $\mathbf{5}$ 's $H_{i}^{(h)}$, which are assigned to $A_{4}$ singlets, transform trivially under $Z_{2}$ and participate in the Yukawa interactions involving charged fermions. Since the remaining three 5's $S_{i}$ are unified into an $A_{4}$ triplet and transform nontrivially under $Z_{2}$, they participate in the Yukawa interactions with the right-handed neutrino $N_{R}$. In analogy to Ref. [26] we assume that the $Z_{2}$ symmetry is not affected by the electroweak symmetry breaking. Therefore, the $A_{4}$ triplet $S_{i}$ does not acquire a VEV and consequently neutrinos do not receive masses at tree-level. The preserved $Z_{2}$ discrete symmetry also allows for stable dark matter candidates, as in Refs. [46, 47]. In our model they are either the lightest neutral component of the $S U(2)$ doublet component of $S_{i}$ or the right-handed Majorana neutrino $N_{R}$. We do not address this question in the present paper. As in Ref. [26], the scalar $\chi$ generates a neutrino mass matrix texture compatible with the experimentally observed deviation from the TBM pattern. As we will explain in the following, the neutrino mass matrix texture generated via the one-loop seesaw mechanism is mainly due to the VEV of this scalar $\langle\chi\rangle=\Lambda_{\text {int }}$, which is assumed to be much larger than the scale of the electroweak symmetry breaking $\Lambda_{i n t} \gg \Lambda_{E W}=246 \mathrm{GeV}$ and at the same time much lower than the GUT scale $\Lambda_{\text {int }} \ll \Lambda_{G U T}=10^{16} \mathrm{GeV}$. This, along with the assumption that the scalars (excepting $\chi$ ) are charged under $U(1)_{f}$, leads to a mixing matrix that is TBM to a good approximation. The $Z_{2}^{\prime}$ discrete symmetry is also an important ingredient of our approach. Once it is imposed, it forbids the terms in the scalar potential involving odd powers of $\chi$. This results in a reduction of the number of free model parameters and selects a particular direction of symmetry breaking in the group space. Also, as will be shown in Sec. IV] due to the $A_{4}$ assignment, the top quark gets its mass mainly from $H^{(3)}$. The $Z_{2}^{\prime}$ symmetry is broken after the $A_{4}$ scalar triplet $\chi$ field acquires a nonvanishing VEV. The symmetry $Z_{2}^{\prime \prime}$ guaranties that the scalars giving the dominant contribution to the masses to the down-type quarks and the charged leptons are different from those providing masses to the up-type quarks. This is crucial for keeping realistic lepton mixing (sf. Ref [26]). The fact that down-type quarks and charged leptons are unified into a $\overline{\mathbf{5}}$ irrep of $S U(5)$ will result in a trivial contribution to the quark mixing from the down-type quark sector. Thus, the quark mixing will arise solely from the up-type quark sector as shown in detail in Sec. IV]

Since the $A_{4}$ triplet $S_{i}$ is assumed to participate in the Yukawa interactions with the right-handed neutrino $N_{R}$, we choose its $U(1)_{f}$ charge $Q_{5}^{(S)}$ to be

$$
Q_{5}^{(S)}=-Q_{\overline{5}}^{(\psi)}
$$


We consider the following VEV pattern of the scalars fields of the model. The VEVs of the scalars $H_{i}^{(h)}(h=1,2,3,4)$, $S_{i}^{(f)}(f=1,2,3)$ and $\Sigma_{j}^{i}$ are

$$
\begin{gathered}
\left\langle H_{i}^{(h)}\right\rangle=v_{H}^{(h)} \delta_{i 5}, \quad\left\langle S_{i}^{(f)}\right\rangle=v_{S}^{(f)} \delta_{i 5}, \quad f=1,2,3, \quad h=1,2,3,4, \\
\left\langle\Sigma_{j}^{i}\right\rangle=v_{\Sigma} \operatorname{diag}\left(1,1,1,-\frac{3}{2},-\frac{3}{2}\right), \quad i, j=1,2,3,4,5 .
\end{gathered}
$$

It is worth mentioning that the VEV pattern for the $\Sigma$ field, which is consistent with the minimization conditions of the scalar potential, follows from the general group theory of spontaneous symmetry breakdown [48].

The requirement that $Z_{2}$ is preserved implies, according to the field assignment given above, that

$$
v_{S}^{(f)}=0, \quad f=1,2,3 .
$$

For the VEVs of the neutral components of the $A_{4}$ triplet scalars $\chi$ and $\xi$ we assume

$$
v_{\chi_{1}}=-v_{\chi_{3}}=\frac{v_{\chi}}{\sqrt{2}}, \quad v_{\chi_{2}}=0, \quad v_{\xi_{1}}=v_{\xi_{2}}=v_{\xi_{3}}=\frac{v_{\xi}}{\sqrt{3}} .
$$

Here $v_{H}^{(h)} \sim \Lambda_{E W}=v=246 \mathrm{GeV}(h=1,2,3)$ and $v_{\chi}=\Lambda_{\text {int }}$. We also assume $v_{\xi}=\Lambda_{G U T}$. The choice of directions in the $A_{4}$ space, given by Eq. (15), is justified by the observation that they describe a natural solution of the scalar potential minimization equations. Indeed, in the single-field case, $A_{4}$ invariance readily favors the $(1,1,1)$ direction over, e.g., the $(1,0,0)$ solution for large regions of parameter space. The vacuum $\langle\xi\rangle$ is a configuration that preserves a $Z_{3}$ subgroup of $A_{4}$, which has been extensively studied by many authors (see for example Refs. 26, 49 57]).

On the other hand, the property of the $\mathbf{4 5}$ dimensional irrep of $S U(5)$ implies that the $\Phi_{j k}^{i}$ satisfies the following relations [30, 31]:

$$
\Phi_{j k}^{i}=-\Phi_{k j}^{i}, \quad \sum_{i=1}^{5} \Phi_{i j}^{i}=0, \quad i, j, k=1,2, \cdots, 5
$$

Consequently, the only allowed nonzero VEVs of $\Phi_{j k}^{i}$ are

$$
\left\langle\Phi_{p 5}^{p}\right\rangle=-\frac{1}{3}\left\langle\Phi_{45}^{4}\right\rangle=v_{\Phi}, \quad\left\langle\Phi_{j 5}^{i}\right\rangle=v_{\Phi}\left(\delta_{j}^{i}-4 \delta_{4}^{i} \delta_{j}^{4}\right), \quad i, j=1,2,3,4,5, \quad \quad p=1,2,3,5 .
$$

With the above particle content, the following renormalizable $\mathcal{L}_{Y}$ and higher-dimensional $\mathcal{L}_{Y}^{(N R)}$ Yukawa terms arise:

$$
\begin{aligned}
& \mathcal{L}_{Y}=\lambda_{\nu}\left(\psi^{i} S_{i}\right)_{\mathbf{1}} N_{R}+M_{N} \bar{N}_{R} N_{R}^{c}+\text { H.c. }, \\
& \mathcal{L}_{Y}^{(N R)}=\frac{\alpha_{1}}{\Lambda}\left(\frac{\Sigma_{l}^{k} \Sigma_{k}^{l}}{\Lambda^{2}}\right)^{a_{1}}\left(\psi^{i} \xi\right)_{\mathbf{1}} H^{j(1)} \Psi_{i j}^{(1)}+\frac{\alpha_{2}}{\Lambda}\left(\frac{\Sigma_{l}^{k} \Sigma_{k}^{l}}{\Lambda^{2}}\right)^{a_{\mathbf{2}}}\left(\psi^{i} \xi\right)_{\mathbf{1}^{\prime \prime}} H^{j(1)} \Psi_{i j}^{(2)}+\frac{\alpha_{3}}{\Lambda}\left(\frac{\Sigma_{l}^{k} \Sigma_{k}^{l}}{\Lambda^{2}}\right)^{a_{3}}\left(\psi^{i} \xi\right)_{\mathbf{1}^{\prime}} H^{j(1)} \Psi_{i j}^{(3)} \\
& +\frac{\beta_{1}}{\Lambda}\left(\frac{\Sigma_{l}^{k} \Sigma_{k}^{l}}{\Lambda^{2}}\right)^{b_{1}}\left(\psi^{i} \xi\right)_{1} \Phi_{i}^{j k} \Psi_{j k}^{(1)}+\frac{\beta_{2}}{\Lambda}\left(\frac{\sum_{l}^{k} \Sigma_{k}^{l}}{\Lambda^{2}}\right)^{b_{2}}\left(\psi^{i} \xi\right)_{1^{\prime \prime}} \Phi_{i}^{j k} \Psi_{j k}^{(2)}+\frac{\beta_{3}}{\Lambda}\left(\frac{\Sigma_{l}^{k} \Sigma_{k}^{l}}{\Lambda^{2}}\right)^{b_{3}}\left(\psi^{i} \xi\right)_{1^{\prime}} \Phi_{i}^{j k} \Psi_{j k}^{(3)} \\
& +\varepsilon^{i j k l p}\left\{\gamma_{12}\left(\frac{\Sigma_{n}^{m} \Sigma_{m}^{n}}{\Lambda^{2}}\right)^{x_{12}} \Psi_{i j}^{(1)} H_{p}^{(3)} \Psi_{k l}^{(2)}+\gamma_{21}\left(\frac{\Sigma_{n}^{m} \Sigma_{m}^{n}}{\Lambda^{2}}\right)^{x_{21}} \Psi_{i j}^{(2)} H_{p}^{(3)} \Psi_{k l}^{(1)}+\gamma_{22}\left(\frac{\Sigma_{n}^{m} \Sigma_{m}^{n}}{\Lambda^{2}}\right)^{x_{22}} \Psi_{i j}^{(2)} H_{p}^{(2)} \Psi_{k l}^{(2)}\right. \\
& +\gamma_{11}\left(\frac{\Sigma_{n}^{m} \sum_{m}^{n}}{\Lambda^{2}}\right)^{x_{11}} \Psi_{i j}^{(1)} H_{p}^{(4)} \Psi_{k l}^{(1)}+\gamma_{23}\left(\frac{\sum_{n}^{m} \Sigma_{m}^{n}}{\Lambda^{2}}\right)^{x_{23}} \Psi_{i j}^{(2)} H_{p}^{(4)} \Psi_{k l}^{(3)}+\gamma_{32}\left(\frac{\sum_{n}^{m} \sum_{m}^{n}}{\Lambda^{2}}\right)^{x_{32}} \Psi_{i j}^{(3)} H_{p}^{(4)} \Psi_{k l}^{(2)} \\
& \left.+\gamma_{13}\left(\frac{\Sigma_{n}^{m} \sum_{m}^{n}}{\Lambda^{2}}\right)^{x_{13}} \Psi_{i j}^{(1)} H_{p}^{(2)} \Psi_{k l}^{(3)}+\gamma_{31}\left(\frac{\sum_{n}^{m} \Sigma_{m}^{n}}{\Lambda^{2}}\right)^{x_{31}} \Psi_{i j}^{(3)} H_{p}^{(2)} \Psi_{k l}^{(1)}+\gamma_{33}\left(\frac{\Sigma_{n}^{m} \Sigma_{m}^{n}}{\Lambda^{2}}\right)^{x_{33}} \Psi_{i j}^{(3)} H_{p}^{(3)} \Psi_{k l}^{(3)}\right\}
\end{aligned}
$$

The subscripts $\mathbf{1}, \mathbf{1}^{\prime}, \mathbf{1}^{\prime \prime}$ denote projecting out the corresponding $A_{4}$ singlet in the product of the two triplets. The lightest of the physical neutral scalar states of $H^{(1)}, H^{(2)}, H^{(3)}, H^{(4)}$ and $\Phi$ should be interpreted as the SM-like 126 GeV Higgs observed at the LHC [58]. Besides that, the low-energy effective theory will correspond to an eight Higgs 
doublet model with three scalar singlets and a light scalar color octet. As we will show in Sec. IV] the dominant contribution to the top quark mass mainly arises from $H^{(3)}$. The SM-like $126 \mathrm{GeV}$ Higgs also receives its main contributions from the $\mathrm{CP}$ even neutral state of the $S U(2)$ doublet part of $H^{(3)}$. The remaining scalars are heavy and outside the LHC reach. Our model is not predictive in the scalar sector, having numerous free uncorrelated parameters in the scalar potential that can be adjusted to get the required pattern of scalar masses. Therefore, the loop effects of the heavy scalars contributing to certain observables can be suppressed by the appropriate choice of the free parameters in the scalar potential. Fortunately, all these adjustments do not affect the charged fermion and neutrino sector, which is completely controlled by the fermion-Higgs Yukawa couplings and by certain combinations of $U(1)_{f}$ charges $Q_{r}^{(i)}$ appearing in the Yukawa terms of Eq. (19). The dimensionless couplings $\alpha_{i}, \beta_{i}$ and $\gamma_{i j}$ $(i, j=1,2,3)$ in Eq. (19) are $\mathcal{O}(1)$ parameters and the following relations for the Froggat-Nielsen powers are fulfilled:

$$
\begin{aligned}
& a_{i}=Q_{\mathbf{1 0}}^{(i)}+Q_{\overline{\mathbf{5}}}^{(\psi)}+Q_{\overline{\mathbf{5}}}^{(1)}+Q_{\mathbf{1}}^{(\xi)}, \\
& b_{i}=Q_{\mathbf{1 0}}^{(i)}+Q_{\overline{\mathbf{5}}}^{(\psi)}-Q_{\mathbf{4 5}}^{(\Phi)}+Q_{\mathbf{1}}^{(\xi)}, \\
& x_{12}=x_{21}=Q_{\mathbf{1 0}}^{(1)}+Q_{\mathbf{1 0}}^{(2)}+Q_{\mathbf{5}}^{(3)} \text {, } \\
& x_{33}=Q_{\mathbf{1 0}}^{(3)}+Q_{\mathbf{1 0}}^{(3)}+Q_{\mathbf{5}}^{(3)} \text {, } \\
& x_{13}=x_{31}=Q_{\mathbf{1 0}}^{(1)}+Q_{\mathbf{1 0}}^{(3)}+Q_{\mathbf{5}}^{(2)} \text {, } \\
& x_{23}=x_{32}=Q_{\mathbf{1 0}}^{(2)}+Q_{\mathbf{1 0}}^{(3)}+Q_{\mathbf{5}}^{(4)} \text {, } \\
& x_{22}=Q_{10}^{(2)}+Q_{10}^{(2)}+Q_{5}^{(2)} \text {, } \\
& x_{11}=Q_{10}^{(1)}+Q_{10}^{(1)}+Q_{5}^{(4)}, \\
& i=1,2,3 \text {. }
\end{aligned}
$$

Furthermore, in order to relate quark masses with the quark mixing parameters, we set

$$
\kappa=\frac{\Sigma_{l}^{k} \Sigma_{k}^{l}}{\Lambda^{2}}=\frac{15 v_{\Sigma}^{2}}{2 \Lambda^{2}}=\frac{\Lambda_{G U T}}{\Lambda}=\lambda .
$$

where $\lambda=0.225$ is one of the parameters in the Wolfenstein parametrization. It is worth mentioning that the terms in the first and second lines of Eq. (19) contribute to the masses of the down-type quarks and charged leptons, while the remaining terms give contributions to the up-type quark masses.

Note that in order to reproduce the nontrivial quark mixing consistent with experimental data, the up-type quark sector requires three 5's, i.e., $H_{i}^{(2)}, H_{i}^{(3)}$ and $H_{i}^{(4)}$ irreps of $S U(5)$ assigned to different $A_{4}$ singlets. In the down-type quark sector, on the other hand, only one $\mathbf{5}$ irrep $H_{i}^{(1)}$, one $\mathbf{4 5}$ irrep $\Phi_{j k}^{i}$ and three $\mathbf{1}$ 's, unified in the $A_{4}$ triplet $\xi=\left(\xi_{1}, \xi_{2}, \xi_{3}\right)$, are needed. As will be shown in the next sections the same set of irreps in the up-type quark sector would lead to the trivial Cabbibo-Kobayashi-Maskawa (CKM) mixing matrix.

\section{LEPTON MASSES AND MIXING}

The charged lepton mass matrix follows from Eq. (19) by using the product rules for the $A_{4}$ group given in Appendix А

$$
M_{l}=\frac{v_{\xi}}{\sqrt{2} \Lambda} V_{l L}^{\dagger}\left(\begin{array}{ccc}
\alpha_{1} \kappa^{a_{1}} v_{H}^{(1)}-6 \beta_{1} \kappa^{b_{1}} v_{\Phi} & 0 & 0 \\
0 & \alpha_{2} \kappa^{a_{2}} v_{H}^{(1)}-6 \beta_{2} \kappa^{b_{2}} v_{\Phi} & 0 \\
0 & 0 & \alpha_{3} \kappa^{a_{3}} v_{H}^{(1)}-6 \beta_{3} \kappa^{b_{3}} v_{\Phi}
\end{array}\right)=V_{l L}^{\dagger} \operatorname{diag}\left(m_{e}, m_{\mu}, m_{\tau}\right),
$$

with

$$
V_{l L}=\frac{1}{\sqrt{3}}\left(\begin{array}{ccc}
1 & 1 & 1 \\
1 & \omega & \omega^{2} \\
1 & \omega^{2} & \omega
\end{array}\right), \quad \omega=e^{\frac{2 \pi i}{3}}
$$

Since we assume that the dimensionless couplings $\alpha_{i}$ and $\beta_{i}(i=1,2,3)$ are roughly of the same order of magnitude and we consider the VEVs $v_{H}^{(1)}$ and $v_{\Phi}$ of the order of the electroweak scale $v \simeq 246 \mathrm{GeV}$, the hierarchy among the charged lepton masses are explained by different combinations of $U(1)_{f}$ charges appearing in the Yukawa terms of Eq. (19).

Since the neutral components of the scalar fields $S_{i}$ have vanishing VEVs, the neutrino mass term does not appear at tree level, as in Ref. [26]. It arises at one-loop level in the form of a Majorana mass term,

$$
-\frac{1}{2} \bar{\nu} M_{\nu} \nu^{C}+\text { H.c. }
$$


from radiative corrections involving the neutral components $H_{i}^{0}$ and $A_{i}^{0}$ of the $S U(2)$ doublet part of $S_{i}$ as well as the heavy Majorana neutrino $N_{R}$ running in the internal lines of the loops. The corresponding diagrams are shown in Fig. 1]
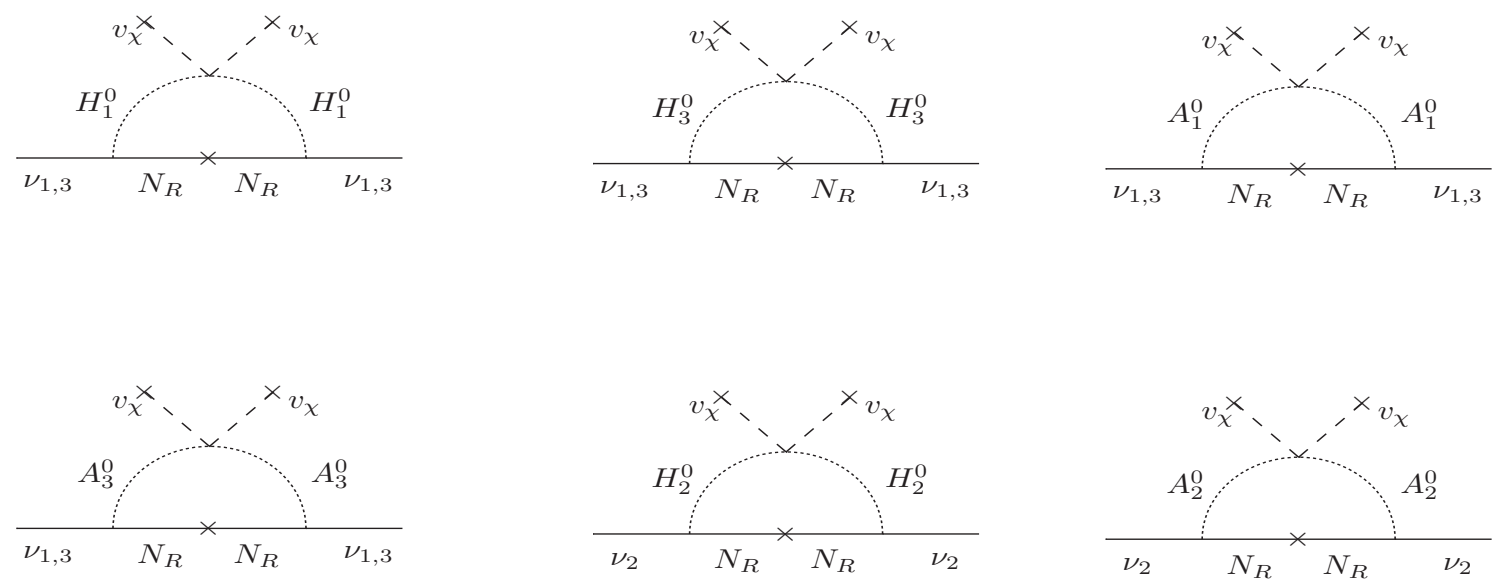

Figure 1: One-loop Feynman diagrams contributing to the entries of the neutrino mass matrix.

Due to the assumption $v_{\chi}>>v$, the quartic scalar interactions relevant for the computation of the neutrino mass matrix are given by the terms

$$
\begin{aligned}
V(S, \chi)= & \lambda_{1}^{(S \chi)}\left(S_{l} S^{l}\right)_{\mathbf{1}}(\chi \chi)_{\mathbf{1}}+\lambda_{2}^{(S \chi)}\left[\left(S_{l} S^{l}\right)_{\mathbf{1}^{\prime}}(\chi \chi)_{\mathbf{1}^{\prime \prime}}+\left(S_{l} S^{l}\right)_{\mathbf{1}^{\prime \prime}}(\chi \chi)_{\mathbf{1}^{\prime}}\right]+\lambda_{3}^{(S \chi)}\left(S_{l} S^{l}\right)_{\mathbf{3} \mathbf{s}}(\chi \chi)_{\mathbf{3} \mathbf{s}} \\
& +\lambda_{4}^{(S \chi)}\left[e^{i \frac{\pi}{2}}\left(S_{l} S^{l}\right)_{\mathbf{3} \mathbf{a}}(\chi \chi)_{\mathbf{3} \mathbf{s}}+H . c .\right] .
\end{aligned}
$$

Following Ref [26] we choose the quartic scalar couplings in the previous expression to be nearly universal, i.e.,

$$
\lambda=\lambda_{2}^{(S \chi)}=\lambda_{3}^{(S \chi)}=\lambda_{1}^{(S \chi)}-\varepsilon .
$$

In practice, the coefficients need not be equal and indeed a nonzero $\varepsilon$ is required to generate two neutrino mass squared differences. Hence, $\varepsilon$ parametrizes the nonuniversality of the relevant couplings. In the approximation described above we obtain the one-loop neutrino mass matrix in the form [26]

$$
M_{\nu} \simeq\left(\begin{array}{ccc}
A e^{2 i \psi} & 0 & A \\
0 & B & 0 \\
A & 0 & A e^{-2 i \psi}
\end{array}\right)
$$

where:

$$
\begin{gathered}
A \simeq \frac{y_{\nu}^{2}}{16 \pi^{2} M_{N}}\left\{\left(M_{A_{1}^{0}}^{2}-M_{A_{2}^{0}}^{2}+\frac{\varepsilon v_{\chi}^{2}}{2}\right)\left[D_{0}\left(\frac{M_{H_{1}^{0}}}{M_{N}}\right)-D_{0}\left(\frac{M_{A_{1}^{0}}}{M_{N}}\right)\right]\right. \\
\left.+\left(M_{A_{3}^{0}}^{2}-M_{A_{2}^{0}}^{2}+\frac{\varepsilon v_{\chi}^{2}}{2}\right)\left[D_{0}\left(\frac{M_{A_{3}^{0}}}{M_{N}}\right)-D_{0}\left(\frac{M_{H_{3}^{0}}}{M_{N}}\right)\right]\right\} \\
B \simeq \frac{\varepsilon y_{\nu}^{2} v_{\chi}^{2}}{16 \pi^{2} M_{N}}\left[D_{0}\left(\frac{M_{H_{2}^{0}}}{M_{N}}\right)-D_{0}\left(\frac{M_{A_{2}^{0}}}{M_{N}}\right)\right] \\
\tan 2 \psi \simeq \frac{1}{\sqrt{\frac{9}{4}\left(\frac{M_{A_{3}^{0}}^{2}-M_{A_{1}^{0}}^{2}}{M_{A_{3}^{0}}^{2}+M_{A_{1}^{0}}^{2}-2 M_{A_{2}^{0}}^{2}}\right)^{2}-1}} .
\end{gathered}
$$


Here $M_{H_{i}^{0}}$ and $M_{A_{i}^{0}}(i=1,2,3)$ are the masses of the CP even and CP odd neutral scalars contained in the $S U(2)$ doublet component of the $S_{i}$. We introduced the function [59]

$$
D_{0}(x)=\frac{-1+x^{2}-\ln x^{2}}{\left(1-x^{2}\right)^{2}} .
$$

As can be seen from Eq. (27) the neutrino mass matrix depends on three effective parameters $A, B$ and $\psi$, which are different combinations of model parameters. To obtain nonvanishing neutrino masses certain requirements need to be fulfilled. To avoid more than one massless neutrino, the universality in the quartic couplings of the scalar potential must be removed, which implies $\varepsilon \neq 0$ or $B \neq 0$. Additionally, to avoid massless neutrinos at one-loop level the masses of the CP-even $H_{i}^{0}$ and CP-odd $A_{i}^{0}$ neutral scalars must be different. This condition implies $A \neq 0$ and $B \neq 0$ as can be seen in Eqs. (28) and (29). The parameters $A$ and $B$ constrain the neutrino mass squared splittings, and the parameter $\psi$ constrains the Pontecorvo-Maki-Nakagawa-Sakata (PMNS) mixing matrix, as will be shown below.

A complex symmetric Majorana mass matrix $M_{\nu}$, as in Eq. (24), can be diagonalized by a unitary rotation of the neutrino fields so that

$$
\nu^{\prime}=V_{\nu} \cdot \nu \quad \longrightarrow \quad V_{\nu}^{\dagger} M_{\nu}\left(V_{\nu}^{\dagger}\right)^{T}=\operatorname{diag}\left(m_{\nu_{1}}, m_{\nu_{2}}, m_{\nu_{3}}\right) \quad \text { with } \quad V_{\nu} V_{\nu}^{\dagger}=\mathbf{1},
$$

where $m_{1,2,3}$ are real and positive. The rotation matrix has the form

$$
V_{\nu}=\left(\begin{array}{ccc}
\cos \theta & 0 & \sin \theta e^{-i \phi} \\
0 & 1 & 0 \\
-\sin \theta e^{i \phi} & 0 & \cos \theta
\end{array}\right) P_{\nu}, \quad \text { with } \quad P_{\nu}=\operatorname{diag}\left(e^{i \alpha_{1} / 2}, e^{i \alpha_{2} / 2}, e^{i \alpha_{3} / 2}\right), \quad \theta= \pm \frac{\pi}{4}, \quad \phi=-2 \psi
$$

We identify the Majorana neutrino masses and Majorana phases $\alpha_{i}$ for the two possible solutions with $\theta=\pi / 4,-\pi / 4$ with $\mathrm{NH}$ and $\mathrm{IH}$, respectively. They are

$$
\begin{aligned}
& \mathrm{NH}: \theta=+\frac{\pi}{4}: \quad m_{\nu_{1}}=0, \quad m_{\nu_{2}}=B, \quad m_{\nu_{3}}=2 A, \quad \alpha_{1}=\alpha_{2}=0, \quad \alpha_{3}=\phi, \\
& \mathrm{IH}: \theta=-\frac{\pi}{4}: \quad m_{\nu_{1}}=2 A, \quad m_{\nu_{2}}=B, \quad m_{\nu_{3}}=0, \quad \alpha_{2}=\alpha_{3}=0, \quad \alpha_{1}=-\phi .
\end{aligned}
$$

Note that the nonvanishing Majorana phases are $\phi$ and $-\phi$ for $\mathrm{NH}$ and $\mathrm{IH}$, respectively.

With the rotation matrices in the charged lepton sector $V_{l L}$, given in Eq. (23), and in the neutrino sector $V_{\nu}$, given in Eq. (32), we find the PMNS mixing matrix:

$$
U=V_{l L}^{\dagger} V_{\nu} \simeq\left(\begin{array}{ccc}
\frac{\cos \theta}{\sqrt{3}}-\frac{e^{i \phi} \sin \theta}{\sqrt{3}} & \frac{1}{\sqrt{3}} & \frac{\cos \theta}{\sqrt{3}}+\frac{e^{-i \phi} \sin \theta}{\sqrt{3}} \\
\frac{\cos \theta}{\sqrt{3}}-\frac{e^{i \phi+\frac{2 i \pi}{3} \sin \theta}}{\sqrt{3}} & \frac{e^{-\frac{2 i \pi}{3}}}{\sqrt{3}} & \frac{e^{\frac{2 i \pi}{3} \cos \theta}}{\sqrt{3}}+\frac{e^{-i \phi} \sin \theta}{\sqrt{3}} \\
\frac{\cos \theta}{\sqrt{3}}-\frac{e^{i \phi-\frac{2 i \pi}{3}} \sin \theta}{\sqrt{3}} & \frac{e^{\frac{2 i \pi}{3}}}{\sqrt{3}} & \frac{e^{-\frac{2 i \pi}{3} \cos \theta}}{\sqrt{3}}+\frac{e^{-i \phi} \sin \theta}{\sqrt{3}}
\end{array}\right) P_{\nu}
$$

It follows from the standard parametrization of the leptonic mixing matrix that the lepton mixing angles are [60]:

$$
\begin{gathered}
\sin ^{2} \theta_{12}=\frac{\left|U_{e 2}\right|^{2}}{1-\left|U_{e 3}\right|^{2}}=\frac{1}{2 \mp \cos \phi}, \quad \sin ^{2} \theta_{13}=\left|U_{e 3}\right|^{2}=\frac{1}{3}(1 \pm \cos \phi), \\
\sin ^{2} \theta_{23}=\frac{\left|U_{\mu 3}\right|^{2}}{1-\left|U_{e 3}\right|^{2}}=\frac{2 \mp(\cos \phi+\sqrt{3} \sin \phi)}{4 \mp 2 \cos \phi},
\end{gathered}
$$

where the upper sign corresponds to $\mathrm{NH}(\theta=+\pi / 4)$ and the lower one to IH $(\theta=-\pi / 4)$. The PMNS matrix (35) of our model reproduces the magnitudes of the corresponding matrix elements of the TBM ansatz (11) in the limit $\phi=0$ $(\mathrm{IH})$ and $\phi=\pi(\mathrm{NH})$ respectively. In both cases the special value for $\phi$ implies that the physical neutral scalars $H_{i}^{0}$ and $A_{i}^{0}$ are degenerate in mass. Notice that the lepton mixing angles are solely controlled by the Majorana phases $\pm \phi$, where the plus and minus signs again correspond to $\mathrm{NH}$ and $\mathrm{IH}$, respectively. 
The Jarlskog invariant $J$ and the CP violating phase $\delta$ are given by [60]:

$$
J=\operatorname{Im}\left(U_{e 1} U_{\mu 2} U_{e 2}^{*} U_{\mu 1}^{*}\right) \simeq-\frac{1}{6 \sqrt{3}} \cos 2 \theta, \quad \sin \delta=\frac{8 J}{\cos \theta_{13} \sin 2 \theta_{12} \sin 2 \theta_{23} \sin 2 \theta_{13}} .
$$

Since $\theta= \pm \frac{\pi}{4}$, we predict $J \simeq 0$ and $\delta \simeq 0$ for $v_{\chi} \gg v$, implying that in our model CP violation is suppressed in neutrino oscillations.

In the following we adjust the free parameters of our model to reproduce the experimental values given in the Tables 【 II and discuss some implications of this choice of the parameters.

As seen from Eqs. (33), (34) and (35), (36) we have only three effective free parameters to fit: $\phi, A$ and $B$. It is noteworthy that in our model a single parameter $(\phi)$ determines all three neutrino mixing parameters $\sin ^{2} \theta_{13}, \sin ^{2} \theta_{12}$ and $\sin ^{2} \theta_{23}$ as well as the Majorana phases $\alpha_{i}$. The parameters $A$ and $B$ control the two mass squared splittings $\Delta m_{i j}^{2}$. Therefore we actually fit only $\phi$ to adjust the values of $\sin ^{2} \theta_{i j}$, while $A$ and $B$ for the NH and the IH hierarchies are simply

$$
\begin{aligned}
& \mathrm{NH}: m_{\nu_{1}}=0, \quad m_{\nu_{2}}=B=\sqrt{\Delta m_{21}^{2}} \approx 9 \mathrm{meV}, \quad m_{\nu_{3}}=2 A=\sqrt{\Delta m_{31}^{2}} \approx 51 \mathrm{meV} ; \\
& \mathrm{IH}: m_{\nu_{2}}=B=\sqrt{\Delta m_{21}^{2}+\Delta m_{13}^{2}} \approx 50 \mathrm{meV}, \quad m_{\nu_{1}}=2 A=\sqrt{\Delta m_{13}^{2}} \approx 49 \mathrm{meV}, \quad m_{\nu_{3}}=0,
\end{aligned}
$$

as follows from Eqs. (33), (34) and the definition $\Delta m_{i j}^{2}=m_{i}^{2}-m_{j}^{2}$. In Eqs. (38), (39) we assumed the best-fit values of $\Delta m_{i j}^{2}$ from Tables [, II.

Varying the model parameter $\phi$ in Eq. (36) we have fitted the $\sin ^{2} \theta_{i j}$ to the experimental values in Tables (1I) The best-fit result is

$$
\begin{aligned}
& \mathrm{NH}: \phi=-0.877 \pi, \quad \sin ^{2} \theta_{12} \approx 0.34, \quad \sin ^{2} \theta_{23} \approx 0.61, \quad \sin ^{2} \theta_{13} \approx 0.0246 \\
& \mathrm{IH}: \phi=0.12 \pi, \quad \sin ^{2} \theta_{12} \approx 0.34, \quad \sin ^{2} \theta_{23} \approx 0.6, \quad \sin ^{2} \theta_{13} \approx 0.025
\end{aligned}
$$

Comparing Eqs. (40), (41) with Tables II II we see that $\sin ^{2} \theta_{13}$ and $\sin ^{2} \theta_{23}$ are in excellent agreement with the experimental data, for both $\mathrm{NH}$ and $\mathrm{IH}$, with $\sin ^{2} \theta_{12}$ within a $2 \sigma$ deviation from its best fit values. It has been shown in Ref. [26] that the solution in Eqs. (38)-(41) does imply neither fine-tuning nor very large values of dimensionful parameters.

With the values of the model parameters given in Eqs. (38)-(41), derived from the oscillation experiments, we can predict the amplitude for neutrinoless double beta $(0 \nu \beta \beta)$ decay, which is proportional to the effective Majorana neutrino mass

$$
m_{\beta \beta}=\sum_{j} U_{e k}^{2} m_{\nu_{k}}
$$

where $U_{e j}^{2}$ and $m_{\nu_{k}}$ are the PMNS mixing matrix elements and the Majorana neutrino masses, respectively.

Then, from Eqs. (32)-(35) and (38)-(41), we predict the following effective neutrino masses for both hierarchies:

$$
m_{\beta \beta}=\frac{1}{3}\left(B+4 A \cos ^{2} \frac{\phi}{2}\right)=\left\{\begin{array}{lll}
4 \mathrm{meV} & \text { for } & \mathrm{NH} \\
50 \mathrm{meV} & \text { for } & \mathrm{IH}
\end{array}\right.
$$

This is beyond the reach of the present and forthcoming $0 \nu \beta \beta$ decay experiments. The presently best upper limit on this parameter $m_{\beta \beta} \leq 160 \mathrm{meV}$ comes from the recently quoted EXO-200 experiment [61] $T_{1 / 2}^{0 \nu \beta \beta}\left({ }^{136} \mathrm{Xe}\right) \geq 1.6 \times 10^{25}$ yr at $90 \%$ C.L. This limit will be improved within a not too distant future. The GERDA experiment 62, 63] is currently moving to "phase-II", at the end of which it is expected to reach $T_{1 / 2}^{0 \nu \beta \beta}\left({ }^{76} \mathrm{Ge}\right) \geq 2 \times 10^{26} \mathrm{yr}$, corresponding to $m_{\beta \beta} \leq 100 \mathrm{meV}$. A bolometric CUORE experiment, using ${ }^{130} \mathrm{Te}$ [64], is currently under construction. Its estimated sensitivity is around $T_{1 / 2}^{0 \nu \beta \beta}\left({ }^{130} \mathrm{Te}\right) \sim 10^{26} \mathrm{yr}$ corresponding to $m_{\beta \beta} \leq 50 \mathrm{meV}$. There are also proposals for ton-scale next-to-next generation $0 \nu \beta \beta$ experiments with ${ }^{136} \mathrm{Xe}[65,66]$ and ${ }^{76} \mathrm{Ge}\left[62,67\right.$ ] claiming sensitivities over $T_{1 / 2}^{0 \nu \beta \beta} \sim 10^{27}$ yr, corresponding to $m_{\beta \beta} \sim 12-30 \mathrm{meV}$. For recent experimental reviews, see for example Ref. [68] and references therein. Thus, according to Eq. (43) our model predicts $T_{1 / 2}^{0 \nu \beta \beta}$ at the level of sensitivities of the next generation or next-to-next generation $0 \nu \beta \beta$ experiments. 


\section{QUARK MASSES AND MIXING}

Using Eq. (19) and the product rules for the $A_{4}$ group listed in Appendix $\mathrm{A}$, we find the mass matrices for up- and down-type quarks in the form

$$
\begin{gathered}
M_{U}=\left(\begin{array}{ccc}
C & F & G \\
F & D & H \\
G & H & E
\end{array}\right), \\
M_{D}=\frac{v_{\xi}}{\sqrt{2} \Lambda}\left(\begin{array}{ccc}
\alpha_{1} \kappa^{a_{1}} v_{H}^{(1)}+2 \beta_{1} \kappa^{b_{1}} v_{\Phi} & 0 \\
0 & \alpha_{2} \kappa^{a_{2}} v_{H}^{(1)}+2 \beta_{2} \kappa^{b_{2}} v_{\Phi} & 0 \\
0 & \alpha_{3} \kappa^{a_{3}} v_{H}^{(1)}+2 \beta_{3} \kappa^{b_{3}} v_{\Phi}
\end{array}\right)\left(V_{l L}^{\dagger}\right)^{T} \\
=\operatorname{diag}\left(m_{d}, m_{s}, m_{b}\right)\left(V_{l L}^{\dagger}\right)^{T},
\end{gathered}
$$

where:

$$
\begin{array}{ll}
F=2\left(\gamma_{12}+\gamma_{21}\right) \kappa^{x_{12}} v_{H}^{(3)}, & D=4 \gamma_{22} \kappa^{x_{22}} v_{H}^{(2)}, \\
G=2\left(\gamma_{13}+\gamma_{31}\right) \kappa^{x_{13}} v_{H}^{(2)}, & C=4 \gamma_{11} \kappa^{x_{11}} v_{H}^{(4)} \\
H=2\left(\gamma_{23}+\gamma_{32}\right) \kappa^{x_{23}} v_{H}^{(4)}, & E=4 \gamma_{33} \kappa^{x_{33}} v_{H}^{(3)}
\end{array}
$$

In analogy to the leptonic sector we assume that the dimensionless couplings $\alpha_{i}, \beta_{i}, \gamma_{i j}(i, j=1,2,3)$ are roughly of the same order of magnitude, with the $\operatorname{VEVs} v_{H}^{(h)}(h=1,2,3,4)$ and $v_{\Phi}$ being at the electroweak scale $v \simeq 246 \mathrm{GeV}$. Then, the hierarchy among the quark masses can be explained by different combinations of $U(1)_{f}$ charges shown in Eq. (20).

The well-known hierarchy among the down-type quark masses is approximately described by

$$
m_{d}: m_{s}: m_{b} \approx \lambda^{4}: \lambda^{2}: 1
$$

with $m_{b} \approx \lambda^{3} m_{t}$

To fulfill the above hierarchy, we set

$$
a_{1}=b_{1}=6, \quad a_{2}=b_{2}=4, \quad a_{3}=b_{3}=2, \quad v_{H}^{(1)} \sim v_{\Phi} \sim \frac{v}{\sqrt{2}} .
$$

Here we have taken into account our previous assumption $v_{\xi}=\lambda \Lambda$ where $\lambda=0.225$ [see Eqs. (15), (21)].

Assuming that the hierarchy of charged fermion masses and quark mixing matrix elements are explained by the Froggatt-Nielsen mechanism we adopt an approximate universality of the dimensionless Yukawa couplings in Eq. (19). Specifically, we set

$$
\beta_{1}=\beta_{3}=-\beta_{2}
$$

so that the down-type quark and charged lepton masses will be determined by four dimensionless parameters, i.e, $\alpha_{1}$, $\alpha_{2}, \alpha_{3}$ and $\beta_{1}$. We fit these parameters to reproduce the experimental values of the down-type quarks and charged leptons. The results are shown in Table III for the following best-fit values of the model parameters:

$$
\alpha_{1}=1.36, \quad \alpha_{2}=2.06, \quad \alpha_{3}=3.77, \quad \beta_{1}=0.18
$$

As customary, we use the quark and charged lepton masses evaluated at the $M_{Z}$ scale [69]. As seen from Table [II there is good agreement of the model values for these masses with the experimental ones.

The CKM quark mixing matrix is defined as 60]

$$
K=R_{U}^{\dagger} R_{D}=\left(\begin{array}{ccc}
K_{u d} & K_{u s} & K_{u b} \\
K_{c d} & K_{c s} & K_{c b} \\
K_{t d} & K_{t s} & K_{t b}
\end{array}\right)
$$




\begin{tabular}{c|c|c}
\hline \hline Observable & Model value & Experimental value \\
\hline$m_{d}(\mathrm{MeV})$ & 2.91 & $2.9_{-0.4}^{+0.5}$ \\
\hline$m_{s}(\mathrm{MeV})$ & 57.1 & $57.7_{-15.7}^{+16.8}$ \\
\hline$m_{b}(\mathrm{GeV})$ & 2.73 & $2.82_{-0.04}^{+0.09}$ \\
\hline$m_{e}(\mathrm{MeV})$ & 0.487 & 0.487 \\
\hline$m_{\mu}(\mathrm{MeV})$ & 102.8 & $102.8 \pm 0.0003$ \\
\hline$m_{\tau}(\mathrm{GeV})$ & 1.75 & $1.75 \pm 0.0003$ \\
\hline
\end{tabular}

Table III: Model and experimental values of the down-type quark and charged lepton masses (at the $M_{Z}$ scale).

where the rotation matrices $R_{D}$ and $R_{U}$ are derived from

$$
R_{U}^{\dagger} M_{U} M_{U}^{\dagger} R_{U}=\operatorname{diag}\left(m_{u}^{2}, m_{c}^{2}, m_{t}^{2}\right), \quad R_{D}^{\dagger} M_{D} M_{D}^{\dagger} R_{D}=\operatorname{diag}\left(m_{d}^{2}, m_{s}^{2}, m_{b}^{2}\right) .
$$

From Eq. (45) it follows that

$$
M_{D} M_{D}^{\dagger}=\operatorname{diag}\left(m_{d}^{2}, m_{s}^{2}, m_{b}^{2}\right) .
$$

Thus $R_{D}=1_{3 \times 3}$ and the CKM quark mixing matrix do not receive contributions from the down-type quark sector, meaning that quark mixing arises solely from the up-type quark sector. Thus, the CKM matrix satisfies the following relation:

$$
M_{U} M_{U}^{\dagger}=K^{\dagger} \operatorname{diag}\left(m_{u}^{2}, m_{c}^{2}, m_{t}^{2}\right) K .
$$

Now we proceed to scan over the parameters of the mass matrix for up-type quarks looking for points where the up-type quark masses, the CKM magnitudes, the Jarlskog invariant and the CP violating phase fit their respective experimental values. In our model the quark CKM matrix is fully determined by the rotation matrix of the up-type quark sector. After scanning the parameter space, we get that a realistic pattern of the quark masses and mixings implies that the mass matrix for up-type quarks should satisfy

$$
M_{U}=m_{t}\left(\begin{array}{ccc}
y \lambda^{10} & f \lambda^{9} e^{i \tau \sigma} & b \lambda^{3} \\
f \lambda^{9} e^{i \tau \sigma} & a \lambda^{4} e^{i \sigma(1+\tau)} & c \lambda^{2} e^{i \sigma} \\
b \lambda^{3} & c \lambda^{2} e^{i \sigma} & d e^{i \sigma}
\end{array}\right),
$$

where $y, a, b, c, d$ and $f$ are $\mathcal{O}(1)$ parameters.

To fulfill these relations, we set

$$
\begin{aligned}
x_{11} & =10, & x_{12}=9, & x_{13}=3 & x_{23}=2, \quad x_{22}=4, & x_{33}=0, \\
v_{H}^{(2)} & \sim v_{H}^{(3)} \sim v_{H}^{(4)} \sim \frac{v}{\sqrt{2}}, & & \left|\gamma_{i j}\right| \sim \frac{1}{4}, & i, j=1,2,3 . &
\end{aligned}
$$

Recall that $\Lambda=\lambda^{-1} \Lambda_{G U T}$ and $\kappa=\lambda$, [see Eqs. (15), (21)].

Therefore, the mass matrix for up-type quarks satisfies the following relation:

$$
\begin{aligned}
M_{U} M_{U}^{\dagger}= & m_{t}^{2}\left(\begin{array}{ccc}
b^{2} \lambda^{6} & b c \lambda^{5} e^{-i \sigma} & b d \lambda^{3} e^{-i \sigma} \\
b c \lambda^{5} e^{i \sigma} & c^{2} \lambda^{4} & c d \lambda^{2} \\
b d \lambda^{3} e^{i \sigma} & c d \lambda^{2} & d^{2}
\end{array}\right) \\
& +m_{t}^{2}\left(\begin{array}{ccc}
\lambda^{18}\left(f^{2}+y^{2} \lambda^{2}\right) & f \lambda^{13}\left(e^{-i \sigma \tau} y \lambda^{6}+a e^{-i \sigma}\right) & \lambda^{11}\left(b y \lambda^{2}+c e^{i \sigma(\tau-1)} f\right) \\
f \lambda^{13}\left(e^{i \sigma \tau} y \lambda^{6}+a e^{i \sigma}\right) & \lambda^{8}\left(f^{2} \lambda^{10}+a^{2}\right) & e^{i \sigma \tau} \lambda^{6}\left(b f \lambda^{6}+a c\right) \\
b y \lambda^{13}+c e^{-i \sigma(\tau-1)} f \lambda^{11} & e^{-i \sigma \tau} \lambda^{6}\left(b f \lambda^{6}+a c\right) & \lambda^{4}\left(c^{2}+b^{2} \lambda^{2}\right)
\end{array}\right),
\end{aligned}
$$

Notice that the first term in Eq. (57) gives the leading contribution to $M_{U} M_{U}^{\dagger}$, while the second one is crucial to generate the up quark mass. 
From Eq. (54) it follows that

$$
M_{U} M_{U}^{\dagger} \simeq m_{t}^{2}\left(\begin{array}{ccc}
\left|K_{t d}\right|^{2} & K_{t d}^{\dagger} K_{t s} & K_{t d}^{\dagger} K_{t b} \\
K_{t s}^{\dagger} K_{t d} & \left|K_{t s}\right|^{2} & K_{t s}^{\dagger} K_{t b} \\
K_{t b}^{\dagger} K_{t d} & K_{t b}^{\dagger} K_{t s} & \left|K_{t b}\right|^{2}
\end{array}\right)
$$

Therefore we can write down

$$
M_{U} M_{U}^{\dagger} \simeq m_{t}^{2}\left(\begin{array}{ccc}
W^{2} \lambda^{6}\left[\eta^{2}+(\rho-1)^{2}\right] & W^{2} \lambda^{5}(-i \eta+\rho-1) & W \lambda^{3}(i \eta-\rho+1) \\
W^{2} \lambda^{5}(i \eta+\rho-1) & W^{2} \lambda^{4} & -W \lambda^{2} \\
W \lambda^{3}(-i \eta-\rho+1) & -W \lambda^{2} & 1
\end{array}\right)
$$

using the Wolfenstein parameterization of the CKM matrix [60, 70]:

$$
K \simeq\left(\begin{array}{ccc}
1-\frac{\lambda^{2}}{2} & \lambda & W \lambda^{3}(\rho-i \eta) \\
-\lambda & 1-\frac{\lambda^{2}}{2} & W \lambda^{2} \\
W \lambda^{3}(1-\rho-i \eta) & -W \lambda^{2} & 1
\end{array}\right)
$$

with the Wolfenstein parameters given by [60]:

$$
\begin{aligned}
& \lambda=0.22535 \pm 0.00065, \quad W=0.811_{-0.012}^{+0.022}, \quad \bar{\rho}=0.131_{-0.013}^{+0.026}, \quad \bar{\eta}=0.345_{-0.014}^{+0.013} \\
& \bar{\rho} \simeq \rho\left(1-\frac{\lambda^{2}}{2}\right), \quad \bar{\eta} \simeq \eta\left(1-\frac{\lambda^{2}}{2}\right) .
\end{aligned}
$$

Comparing Eqs. (57) and (59) we find the following relations:

$$
b \simeq W \sqrt{\eta^{2}+(\rho-1)^{2}}, \quad c \simeq-W, \quad d \simeq 1, \quad \sigma \simeq \arctan \left(-\frac{\eta}{1-\rho}\right) .
$$

Since $d \simeq 1$, it follows from the previous relations that the quark mixing in our model is described by five effective dimensionless parameters, i.e., $b, c, \sigma, \tau$ and $\lambda$. The $\lambda$ parameter in the Wolfenstein parametrization is fixed by the ratio between the grand unification scale $\Lambda_{G U T}$ and the cutoff $\Lambda$ of our model.

We fit the remaining $\mathcal{O}(1)$ parameters in Eq. (55) to reproduce the up-type quark mass spectrum and quark mixing parameters. The results are shown in Table IV for the following best-fit values:

$$
y=0.2, \quad f=0.39, \quad a=0.26, \quad \tau=2.9 .
$$

The CKM matrix in our model is in excellent agreement with the experimental data. The agreement of our model with the experimental data is as good as in the models of Refs. [71 76] and better than, for example, those in Refs. [77 83]. The values of these observables as well as the up-type quark masses are juxtaposed together with the experimental data in Table IV] The experimental values of the quark masses, which are given at the $M_{Z}$ scale, have been taken from Ref. 69], whereas the experimental values of the CKM matrix elements and the Jarlskog invariant $J$ are taken from Ref. [60]. As seen from Table [V] all the analyzed physical parameters are in very good agreement with the experimental data, except for $m_{u}$, and $m_{c}$, which reproduce the corresponding experimental values only with order of magnitude accuracy.

\section{CONCLUSIONS}

We proposed a model based on the group $S U(5) \otimes A_{4} \otimes Z_{2} \otimes Z_{2}^{\prime} \otimes Z_{2}^{\prime \prime} \otimes U(1)_{f}$, which is an extension of the model of Ref. [26]. The model has in total 14 effective free parameters, which allowed us to reproduce 18 observables, i.e., 9 charged fermion masses, 2 neutrino mass squared splittings, 3 lepton mixing parameters and the 4 parameters of the Wolfenstein parametrization of the CKM quark mixing matrix. The observed hierarchy of the charged fermion masses arises from a generalized Froggatt-Nielsen mechanism where the charged fermions get masses via nonrenormalizable operators invariant under the gauge and flavor symmetries. It is triggered by a scalar field $\Sigma$ in the $\mathbf{2 4}$ representation 


\begin{tabular}{c|c|c}
\hline \hline Observable & Model value & Experimental value \\
\hline$m_{u}(\mathrm{MeV})$ & 5.4 & $1.45_{-0.45}^{+0.56}$ \\
\hline$m_{c}(\mathrm{MeV})$ & 284 & $635 \pm 86$ \\
\hline$m_{t}(\mathrm{GeV})$ & 173.4 & $172.1 \pm 0.6 \pm 0.9$ \\
\hline$\left|V_{u d}\right|$ & 0.974 & $0.97427 \pm 0.00015$ \\
\hline$\left|V_{u s}\right|$ & 0.225 & $0.22534 \pm 0.00065$ \\
\hline$\left|V_{u b}\right|$ & 0.00348 & $0.00351_{-0.00014}^{+0.00015}$ \\
\hline$\left|V_{c d}\right|$ & 0.225 & $0.22520 \pm 0.00065$ \\
\hline$\left|V_{c s}\right|$ & 0.973 & $0.97344 \pm 0.00016$ \\
\hline$\left|V_{c b}\right|$ & 0.0422 & $0.0412_{-0.0005}^{+0.0011}$ \\
\hline$\left|V_{t d}\right|$ & 0.00872 & $0.00867_{-0.00031}^{+0.00029}$ \\
\hline$\left|V_{t s}\right|$ & 0.0415 & $0.0404_{-0.0005}^{+0.0011}$ \\
\hline$\left|V_{t b}\right|$ & 0.999 & $0.999146_{-0.000046}^{+0.000021}$ \\
\hline$J$ & $2.95 \times 10^{-5}$ & $\left(2.96_{-0.16}^{+0.20}\right) \times 10^{-5}$ \\
\hline$\delta$ & $66^{\circ}$ & $68^{\circ}$ \\
\hline \hline
\end{tabular}

Table IV: Model and experimental values of the up-type quark masses and CKM parameters.

of $S U(5)$ charged under the global $U(1)_{f}$ symmetry and acquiring a VEV at the GUT scale. Thus, the hierarchy of the charged fermion masses and the quark mixing matrix elements arises as a consequence of the power dependence of the charged fermion mass matrix elements on particular combinations of the $U(1)_{f}$ charges.

The neutrino masses in our model arise from a radiative seesaw mechanism, which explains their smallness, while keeping the mass of the right-handed neutrino at comparatively low values, which could be about a few TeV. The neutrino mixing is approximately tribimaximal due to the spontaneously broken $A_{4}$ symmetry of the model. The experimentally observed deviation from the TBM pattern is implemented by introducing the $S U(5)$ singlet $A_{4}$ triplet $\chi$. Its VEV $\langle\chi\rangle \gg \Lambda_{E W}$ properly shapes the neutrino mass matrix at the one-loop level. The model predicts strong suppression of the $\mathrm{CP}$ violation in neutrino oscillations.

The predicted values of the effective Majorana neutrino mass $m_{\beta \beta}$ for $0 \nu \beta \beta$ decay are 4 and $50 \mathrm{meV}$ for the normal and the inverted neutrino spectrum, respectively.

An unbroken $Z_{2}$ discrete symmetry of our model also allows for stable dark matter candidates, as in Refs. [46, 47]. They could be either the lightest neutral component of the $S U(5) 5$-plet $S_{i}$ or the right-handed Majorana neutrino $N_{R}$. We do not address this subject in the present paper.

\section{Acknowledgments}

A.E.C.H. was partially supported by Fondecyt (Chile), Grants No. 11130115, No. 1100582 and No. 1140390 and by DGIP internal Grant No. 111458. A.E.C.H thanks Professor Stefan Antusch and Dr. Ivo de Mendeiros Varzielas for useful criticism. E.S. was supported by DFG CONICYT Grant No. PA 803/7-1. E.S. acknowledges the hospitality at UTFSM during part of this collaboration.

\section{Appendix A: The product rules for $A_{4}$}

The following product rules for the $A_{4}$ group were used in the construction of our model Lagrangian:

$$
\mathbf{3} \otimes \mathbf{3}=\mathbf{3}_{s} \oplus \mathbf{3}_{a} \oplus \mathbf{1} \oplus \mathbf{1}^{\prime} \oplus \mathbf{1}^{\prime \prime},
$$

$$
1 \otimes 1=1, \quad 1^{\prime} \otimes 1^{\prime \prime}=1, \quad 1^{\prime} \otimes 1^{\prime}=1^{\prime \prime}, \quad 1^{\prime \prime} \otimes 1^{\prime \prime}=1^{\prime} .
$$

Denoting $\left(x_{1}, y_{1}, z_{1}\right)$ and $\left(x_{2}, y_{2}, z_{2}\right)$ as the basis vectors for two $A_{4}$-triplets $\mathbf{3}$, one finds 


$$
\begin{aligned}
& (\mathbf{3} \otimes \mathbf{3})_{\mathbf{1}}=x_{1} y_{1}+x_{2} y_{2}+x_{3} y_{3}, \\
& (\mathbf{3} \otimes \mathbf{3})_{\mathbf{3}_{s}}=\left(x_{2} y_{3}+x_{3} y_{2}, x_{3} y_{1}+x_{1} y_{3}, x_{1} y_{2}+x_{2} y_{1}\right), \quad(\mathbf{3} \otimes \mathbf{3})_{\mathbf{1}^{\prime}}=x_{1} y_{1}+\omega x_{2} y_{2}+\omega^{2} x_{3} y_{3}, \\
& (\mathbf{3} \otimes \mathbf{3})_{\mathbf{3}_{a}}=\left(x_{2} y_{3}-x_{3} y_{2}, x_{3} y_{1}-x_{1} y_{3}, x_{1} y_{2}-x_{2} y_{1}\right), \quad(\mathbf{3} \otimes \mathbf{3})_{\mathbf{1}^{\prime \prime}}=x_{1} y_{1}+\omega^{2} x_{2} y_{2}+\omega x_{3} y_{3},
\end{aligned}
$$

where $\omega=e^{i \frac{2 \pi}{3}}$. The representation $\mathbf{1}$ is trivial, while the nontrivial $\mathbf{1}^{\prime}$ and $\mathbf{1}^{\prime \prime}$ are complex conjugate to each other. Comprehensive reviews of discrete symmetries in particle physics can be found in Refs. [24, 84 86].

[1] S. F. King, A. Merle, S. Morisi, Y. Shimizu and M. Tanimoto, New J. Phys. 16, 045018 (2014) arXiv:1402.4271 [hep-ph]].

[2] G. Altarelli and F. Feruglio, Springer Tracts Mod. Phys. 190, 169 (2003) hep-ph/0206077.

[3] H. Fritzsch and Z. -z. Xing, Prog. Part. Nucl. Phys. 45, 1 (2000) hep-ph/9912358.

[4] F. P. An et al. [DAYA-BAY Collaboration], Phys. Rev. Lett. 108, 171803 (2012) [arXiv:1203.1669 [hep-ex]].

[5] K. Abe et al. [T2K Collaboration], Phys. Rev. Lett. 107, 041801 (2011) arXiv:1106.2822 [hep-ex]].

[6] P. Adamson et al. [MINOS Collaboration], Phys. Rev. Lett. 107, 181802 (2011) arXiv:1108.0015 [hep-ex]].

[7] Y. Abe et al. [DOUBLE-CHOOZ Collaboration], Phys. Rev. Lett. 108, 131801 (2012) arXiv:1112.6353 [hep-ex]].

[8] J. K. Ahn et al. [RENO Collaboration], Phys. Rev. Lett. 108, 191802 (2012) arXiv:1204.0626 [hep-ex]].

[9] D. V. Forero, M. Tortola and J. W. F. Valle, Phys. Rev. D 86, 073012 (2012) arXiv:1205.4018 [hep-ph]].

[10] D. Marzocca, S. T. Petcov, A. Romanino and M. Spinrath, JHEP 1111, 009 (2011) arXiv:1108.0614 [hep-ph]].

[11] S. Antusch, C. Gross, V. Maurer and C. Sluka, Nucl. Phys. B 877, 772 (2013) [arXiv:1305.6612 [hep-ph]].

[12] M. -C. Chen, J. Huang, K. T. Mahanthappa and A. M. Wijangco, JHEP 1310, 112 (2013) arXiv:1307.7711 [hep-ph]].

[13] S. F. King, C. Luhn and A. J. Stuart, Nucl. Phys. B 867, 203 (2013) arXiv:1207.5741 [hep-ph]].

[14] D. Meloni, JHEP 1110, 010 (2011) arXiv:1107.0221 [hep-ph]].

[15] P. S. Bhupal Dev, B. Dutta, R. N. Mohapatra and M. Severson, Phys. Rev. D 86, 035002 (2012) [arXiv:1202.4012 [hep-ph]].

[16] K. S. Babu and Y. Meng, Phys. Rev. D 80, 075003 (2009) arXiv:0907.4231 [hep-ph]].

[17] K. S. Babu, K. Kawashima and J. Kubo, Phys. Rev. D 83, 095008 (2011) arXiv:1103.1664 [hep-ph]].

[18] J. C. Gómez-Izquierdo, F. G. 'al. Canales and M. Mondragón, arXiv:1312.7385 [hep-ph].

[19] S. Antusch, S. F. King and M. Spinrath, Phys. Rev. D 83, 013005 (2011) arXiv:1005.0708 [hep-ph]].

[20] C. Hagedorn, S. F. King and C. Luhn, JHEP 1006, 048 (2010) arXiv:1003.4249 [hep-ph]].

[21] H. Ishimori, Y. Shimizu and M. Tanimoto, Prog. Theor. Phys. 121, 769 (2009) [arXiv:0812.5031 [hep-ph]].

[22] K. M. Patel, Phys. Lett. B 695, 225 (2011)

[23] M. -C. Chen and K. T. Mahanthappa, Int. J. Mod. Phys. A 18, 5819 (2003) hep-ph/0305088.

[24] S. F. King and C. Luhn, Rept. Prog. Phys. 76, 056201 (2013) arXiv:1301.1340 [hep-ph]].

[25] F. Wang and Y. -X. Li, Eur. Phys. J. C 71, 1803 (2011) arXiv:1103.6017 [hep-ph]].

[26] A. E. Cárcamo Hernández, I. d. M. Varzielas, S. G. Kovalenko, H. Päs and I. Schmidt, Phys. Rev. D 88, 076014 (2013) arXiv:1307.6499 [hep-ph]].

[27] E. Ma, Phys. Rev. Lett. 81, 1171 (1998) hep-ph/9805219.

[28] Y. H. Ahn, S. K. Kang and C. S. Kim, Phys. Rev. D 87, 113012 (2013) arXiv:1304.0921 [hep-ph]].

[29] H. Georgi and S. L. Glashow, Phys. Rev. Lett. 32, 438 (1974).

[30] H. Georgi and C. Jarlskog, Phys. Lett. B 86, 297 (1979).

[31] P. Frampton, S. Nandi and J. Scanio, Phys. Lett. B 85, 225 (1979).

[32] J. R. Ellis and M. K. Gaillard, Phys. Lett. B 88, 315 (1979).

[33] S. Nandi and K. Tanaka, Phys. Lett. B 92, 107 (1980).

[34] P. H. Frampton, Phys. Lett. B 89, 352 (1980).

[35] P. Langacker, Phys. Rept. 72, 185 (1981).

[36] P. Kalyniak and J. N. Ng, Phys. Rev. D 26, 890 (1982).

[37] A. Giveon, L. J. Hall and U. Sarid, Phys. Lett. B 271, 138 (1991).

[38] I. Dorsner and I. Mocioiu, Nucl. Phys. B 796, 123 (2008) arXiv:0708.3332 [hep-ph]].

[39] I. Dorsner and P. Fileviez Perez, Phys. Lett. B 642, 248 (2006) hep-ph/0606062.

[40] P. Fileviez Pèrez, In *Karlsruhe 2007, SUSY 2007*678-681 arXiv:0710.1321 [hep-ph]].

[41] P. Fileviez Pèrez, H. Iminniyaz and Germán Rodrigo, Phys. Rev. D 78, 015013 (2008) arXiv:0803.4156 [hep-ph]].

[42] S. Khalil and S. Salem, Nucl. Phys. B 876, 473 (2013) arXiv:1304.3689 [hep-ph]].

[43] H. Ishimori and E. Ma, Phys. Rev. D 86, 045030 (2012) arXiv:1205.0075 [hep-ph]].

[44] S. Morisi, D. V. Forero, J. C. Romao and J. W. F. Valle, Phys. Rev. D 88, 016003 (2013) arXiv:1305.6774 [hep-ph]].

[45] P. Binetruy, Supersymmetry: Theory, Experiment and Cosmology, Oxford University Press, UK (2007).

[46] E. Ma, Phys. Rev. D 73, 077301 (2006) hep-ph/0601225].

[47] E. Ma, Mod. Phys. Lett. A 211777 (2006) hep-ph/0605180].

[48] L. -F. Li, Phys. Rev. D 9, 1723 (1974).

[49] E. Ma, H. Sawanaka and M. Tanimoto, Phys. Lett. B 641, 301 (2006) hep-ph/0606103. 
[50] G. Altarelli and F. Feruglio, Nucl. Phys. B 72064 (2005) hep-ph/0504165].

[51] G. Altarelli and F. Feruglio, Nucl. Phys. B 741215 (2006) hep-ph/0512103.

[52] X. -G. He, Y. -Y. Keum and R. R. Volkas, JHEP 0604039 (2006) hep-ph/0601001].

[53] N. Memenga, W. Rodejohann and H. Zhang, Phys. Rev. D 87 (2013) 053021 [arXiv:1301.2963 [hep-ph]].

[54] R. de Adelhart Toorop, F. Bazzocchi, L. Merlo and A. Paris, JHEP 1103, 035 (2011) [Erratum-ibid. 1301, 098 (2013)] arXiv:1012.1791 [hep-ph]].

[55] Y. H. Ahn and S. K. Kang, Phys. Rev. D 86093003 (2012) arXiv:1203.4185 [hep-ph]].

[56] R. N. Mohapatra and C. C. Nishi, Phys. Rev. D 86073007 (2012) arXiv:1208.2875 [hep-ph]].

[57] M. -C. Chen, J. Huang, J. -M. O'Bryan, A. M. Wijangco and F. Yu, JHEP 1302021 (2013) arXiv:1210.6982 [hep-ph]].

[58] G. Aad et al. [ATLAS Collaboration], Phys. Lett. B 716, 1 (2012); S. Chatrchyan et al. [CMS Collaboration], Phys. Lett. B 716, 30 (2012).

[59] A. E. Cárcamo Hernández, R. Martínez and F. Ochoa, Phys. Rev. D 87075009 (2013) arXiv:1302.1757 [hep-ph]].

[60] J. Beringer et al. (Particle Data Group), Phys. Rev. D 86010001 (2012).

[61] EXO Collaboration, M. Auger et al., Phys.Rev.Lett. 109, 032505 (2012), arXiv:1205.5608.

[62] GERDA Collaboration, I. Abt et al., (2004), arXiv:hep-ex/0404039.

[63] GERDA Collaboration, K.-H. Ackermann et al., (2012), Eur. Phys. J. C 73, 2330 (2013) arXiv:1212.4067[physics.ins-det]].

[64] F. Alessandria et al., (2011), arXiv:1109.0494,

[65] KamLAND-Zen Collaboration, A. Gando et al., Phys.Rev. C85, 045504 (2012), arXiv:1201.4664.

[66] EXO-200 Collaboration, D. Auty, Recontres de Moriond, http://moriond.in2p3.fr/ (2013).

[67] Majorana Collaboration, C. Aalseth et al., Nucl.Phys.Proc.Suppl. 217, 44 (2011), arXiv:1101.0119.

[68] A. S. Barabash, arXiv:1209.4241 [nucl-ex].

[69] K. Bora, arXiv:1206.5909 [hep-ph].

[70] L. Wolfenstein, Phys. Rev. Lett. 51, 1945 (1983).

[71] S. F. King, S. Morisi, E. Peinado and J. W. F. Valle, Phys. Lett. B 724, 68 (2013) arXiv:1301.7065 [hep-ph]].

[72] A. E. Cárcamo Hernández and R. Rahman, arXiv:1007.0447 [hep-ph].

[73] G. Bhattacharyya, I. de Medeiros Varzielas and P. Leser, Phys. Rev. Lett. 109, 241603 (2012) [arXiv:1210.0545 [hep-ph]].

[74] A. E. Cárcamo Hernández, R. Martinez and F. Ochoa, arXiv:1309.6567 [hep-ph].

[75] A. E. Cárcamo Hernández, R. Martinez and Jorge Nisperuza, arXiv:1401.0937 [hep-ph].

[76] A. E. Cárcamo Hernández and I. de Medeiros Varzielas, to appear.

[77] H. Fritzsch, Phys. Lett. B 70, 436 (1977), Phys. Lett. B 73, 317 (1978), Nucl. Phys. B 155, 189 (1979); H. Fritzsch and J. Planck, Phys. Lett. B 237, 451 (1990).

[78] D. s. Du and Z. z. Xing, Phys. Rev. D 48, 2349 (1993);

[79] H. Fritzsch and Z. z. Xing, Phys. Lett. B 353, 114 (1995) arXiv:hep-ph/9502297, Nucl. Phys. B 556, 49 (1999) arXiv:hep-ph/9904286, Prog. Part. Nucl. Phys. 45, 1 (2000) arXiv:hep-ph/9912358, Phys. Lett. B 555, 63 (2003) arXiv:hep-ph/0212195.

[80] H. Nishiura, K. Matsuda, T. Kikuchi and T. Fukuyama, Phys. Rev. D 65, 097301 (2002) arXiv:hep-ph/0202189; K. Matsuda and H. Nishiura, Phys. Rev. D 69, 053005 (2004) arXiv:hep-ph/0309272.

[81] Y. F. Zhou, J. Phys. G 30, 783 (2004) arXiv:hep-ph/0307240.

[82] A. E. Cárcamo Hernández, R. Martinez and J. A. Rodriguez, Eur. Phys. J. C 50, 935 (2007) arXiv:hep-ph/0606190, AIP Conf. Proc. 1026 (2008) 272.

[83] A. E. Cárcamo Hernández, C. O. Dib, N. Neill H and A. R. Zerwekh, JHEP 1202, 132 (2012) [arXiv:1201.0878 [hep-ph]].

[84] G. Altarelli and F. Feruglio, Rev. Mod. Phys. 822701 (2010) arXiv:1002.0211 [hep-ph]].

[85] H. Ishimori, T. Kobayashi, H. Ohki, Y. Shimizu, H. Okada and M. Tanimoto, Prog. Theor. Phys. Suppl. 183, 1 (2010) arXiv:1003.3552 [hep-th]].

[86] P. Ramond, Group Theory: A Physicist's Survey, Cambridge University Press, UK (2010). 